\title{
Relationship between periodontal disease (periodontitis) and acute vascular events a case- control study enperiva study
}

\begin{abstract}
Introduction and objectives: Periodontal disease (periodontitis) and atheromatous disease share not only risk factors such as smoking and diabetes, but also a complex multifactorial process of chronic inflammation stimulating mediators that accelerate endothelial dysfunction and/or mechanisms of cross-reacting antibodies against the periodontal flora and endothelial cell proteins; this may explain the association between both pathologies. The aim of our study was to add evidence about the relationship between periodontal disease (periodontitis) and vascular disease, using acute vascular events as the primary endpoint.
\end{abstract}

Methods: A case-control study was conducted. A total of 30 cases and 32 controls were included in a non-randomized selection.

Results: The presence of periodontal disease was significantly higher in cases than it was in controls ( $70 \%$ vs. $40 \%$; OR [ $95 \% \mathrm{CI}$ ]: 3.41 [1.19-9.76]; p=0.02). Also, plaque and gingival indexes were significantly higher in cases (PI: $1.74 \pm 0.64$ vs. $0.99 \pm 0.69$, respectively, $\mathrm{p}<0.01$; GI: $1.61 \pm 0.66$ vs. $0.98 \pm 0.55$, respectively, $\mathrm{p}<0.01$ ).

Conclusion: This study demonstrates a strong association between periodontal disease and acute vascular events, the former having an even greater impact than traditional risk factors.
Volume 7 Issue 5 - 2017

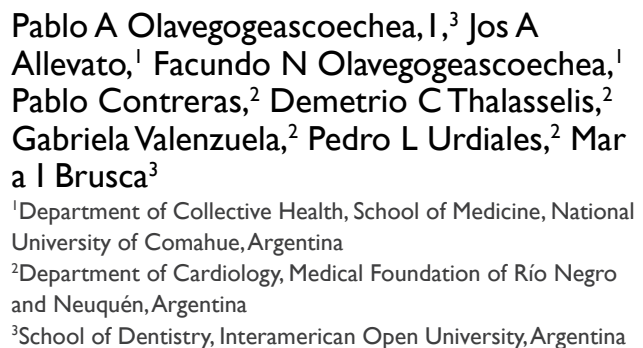

${ }^{3}$ School of Dentistry, Interamerican Open University, Argentina

Correspondence: Pablo A Olavegogeascoechea, Collective Health Department, School of Medicine, National University of Comahue, Cipolletti, Luis Toschi avenue and Arrayanes street, Cipolletti (8324), Río Negro, Argentina,

Email polavego@gmail.com

Received: July 01, 2017 | Published: July 21, 2017

Keywords: periodontal disease, periodontitis, acute vascular events, acute coronary syndrome

\section{Introduction}

Periodontal disease(POD) is characterized by infection and chronic inflammation. This condition starts with the presence of bacteria in the gingival sulcus (e.g. Streptococcus sanguis, Streptococcus oralis, Actinomyces odontolyticus, Fusobacterium nucleatum) followed by a chronic inflammation process affecting the periodontal pocket apically and leading to the destruction of the tooth-supporting structure $^{1}$. Besides the typical risk factors (RF) for POD (smoking, diabetes, obesity), a complex mechanism involving these factors along with genetic factors and infection have been found to play a role. ${ }^{2}$ The result of chronic inflammation can be determined through clinical and radiographic parameters as well as plasma and gingival fluid analysis..$^{3-6}$ On the other hand, vascular disease and the events associated with it are one of the leading causes of morbidity and mortality in adults..$^{7-8}$ In this context, atheromatous disease constitutes the most common cause of vascular events, with plaque complications being the reason for most acute episodes. Persistent inflammation may explain the pathogenesis, progression and, finally, the rupture of the atheromatous plaque. ${ }^{9-10}$ The inflammatory state is triggered and sustained by the vascular disease risk factors first described in the Framingham Study. ${ }^{11}$ However, not all vascular events were related to those RF; during the last 15 years, POD has started to be regarded as a risk factor strongly associated with atheromatous disease. ${ }^{1-12}$ Periodontal disease and atheromatous disease share not only risk factors such as smoking and diabetes, but also a complex multifactorial process of chronic inflammation stimulating mediators that accelerate endothelial dysfunction and/or mechanisms of cross- reacting antibodies against the periodontal flora and endothelial cell proteins; this may explain the relation between these pathologies. ${ }^{13-15}$ The studies which have explored the association between these two diseases were published with different biases as regards the endpoints to determine this relation, ${ }^{16}$ the design, the population, the sample size and even the different definitions of periodontal disease. ${ }^{17,18}$ The aim of our study was to establish the relation between periodontal disease (periodontitis) and vascular disease, using acute vascular events (AVE) as the primary endpoint.

\section{Materials and methods}

A case-control study was conducted from March to August 2016. The subjects who participated were enrolled in only one institution. The selection of cases was non-randomized; the participants were patients suffering an acute vascular event such as: acute coronary syndrome (ACS), ischemic stroke (IschS) or acute peripheral artery disease/acute limb ischemia (PAD), and all admitted to the cardiac intensive care unit. Dental evaluation was carried out within $72 \pm 24.8$ hours after admission. Controls were included based on a 1:1 casecontrol ratio, except for the two last cases, for which a 1:2 ratio was used, and they were paired according to age ( \pm 5 years), gender and typical risk factors, i.e. hypertension, diabetes, dyslipidemia, family history of coronary artery disease, sedentary lifestyle, current or former smoking habit and BMI $\left( \pm 3 \mathrm{~kg} / \mathrm{m}^{3}\right)$. Given that most patients were exposed to more than one risk factor for vascular disease, the matching was based on those risk factors which have a greater probability of precipitating vascular events according to the 
Framingham risk score (high blood pressure, diabetes, smoking). ${ }^{19} \mathrm{~A}$ total number of 36 potential participants were recruited; 2 of them refused to participate, 3 were discharged from hospital before dental evaluation and 1 before blood sampling; 32 controls were included. The screening of controls and cases was done contemporaneously; the average interval between the enrollment of a case and the enrollment of its corresponding control was $2.3 \pm 0.7$ weeks. The study was approved by the Research Department and the Ethics Committee at Universidad Abierta Interamericana (Interamerican Open University) (PS2/2014) and conducted in accordance with the 1975 Declaration of Helsinki, revised in 1983 and 2013. Before enrolling, participants had to sign an informed consent.

\section{Definitions}

I. We define ACS as: (unstable angina, non-ST segment elevation $\mathrm{MI},{ }^{20}$ or ST segment elevation $\mathrm{MI}$ ):

a. Unstable angina (UA) is defined as ischemic chest discomfort (or equivalent) that occurs at rest with at least 1 episode lasting $\geq 10$ minutes and is accompanied by new or presumably new ST segment deviation (transient $[<20$ minutes] elevation $\geq 0.1 \mathrm{mV}$ or dynamic horizontal/down-sloping depression $\geq 0.05 \mathrm{mV}$ ) in at least 2 contiguous leads without diagnostic biochemical changes in cardiac enzymes (serum troponin I or T, or creatine kinase-MB).

b. Non-ST segment elevation MI (NSTEMI) is defined as ischemic chest discomfort (or equivalent) that occurs at rest with at least 1 episode lasting $\geq 10$ minutes and is accompanied by a diagnostic elevation in cardiac biomarkers of myocardial injury (serum troponin I or T, or creatine kinase-MB) above the upper limit of normal without persistent ST segment elevation.

c. ST segment elevation MI (STEMI) is defined as prolonged symptoms of ischemic chest discomfort (or equivalent) at rest (with at least 1 episode lasting $>20 \mathrm{~min}$ ) and new or presumably new electrocardiographic changes (persistent ST segment elevation $\geq 0.1 \mathrm{mV}$ in $\geq 2$ contiguous precordial leads or $\geq 2$ adjacent limb leads or new LBBB) that are accompanied by a diagnostic elevation in cardiac biomarkers (serum troponin I or $\mathrm{T}$, creatine kinase or creatine kinase-MB) above the upper limit of normal.

II. We define Ischemic Stroke as: documented focal neurologic deficit thought to be of vascular origin, with signs or symptoms lasting $>24$ hours. Diagnosis was confirming by neuroimaging, such as computed tomography (CT) scan or magnetic resonance imaging (MRI). The embolic origin was discarded as the cause of ischemia.

III. Acute peripheral artery disease (APAD) was documented by one of the following: current intermittent claudication with objective evidence of vascular origin, history of peripheral arterial stenting or surgery (including amputation due to vascular causes), or ankle-brachial index $<0.9$ in at least one ankle and the onset of acute limb pain followed by coldness and numbness of the right foot confirmed by angiography. The embolic origin was discarded as the cause of ischemia.

IV. Risk factors for vascular disease: family history $(\mathrm{FH})$ of acute ischemic heart disease(any first degree relative [parents, siblings, children] who have had angina, myocardial infarction or sudden death with no apparent cause before the age of 55), diabetes mellitus (history of diabetes, need for oral antidiabetic drugs or insulin use or fasting blood glucose $>110 \mathrm{mg} / \mathrm{dL}$ ), high blood pressure (HBP) (history of high blood pressure diagnosed and treated with medication, exercise and/or diet; systolic blood pressure $>140 \mathrm{mmHg}$ or diastolic blood pressure $>90 \mathrm{mmHg}$ at least twice; current use of antihypertensive medication), being a smoker (having smoked in the month before admission or having stopped smoking between 1 month and 1 year before inclusion) or a former smoker (having stopped $>1$ year before inclusion), overweight/obesity $\left(\mathrm{BMI}>25 \mathrm{~kg} / \mathrm{m}^{3}\right.$ ), dyslipidemia (history of dyslipidemia diagnosed and/or being treated with hypolipemic agents), sedentary lifestyle (individuals who engage in lowintensity physical activity). ${ }^{21}$

Inclusion criteria: Signature on the informed consent for both groups.

a. Cases: patients admitted to the intensive care unit with ACS, APAD and/or ischemic stroke aged $>21$; patients who have not taken vitamin or mineral supplements in the last 3 months; presence of at least 7 teeth.

b. Controls: patients admitted to the intensive care unit or in the general ward without acute vascular events, aged $>21$; patients who have not taken vitamin or mineral supplements in the last 3 months; presence of at least 7 teeth.

Exclusion criteria: patients with cancer of any origin previously diagnosed; patients with cirrhosis, known biliary abnormalities (with the exception of Gilbert's syndrome or asymptomatic gallstones), unstable liver disease, or evidence of abnormal liver function tests (total bilirubin or alkaline phosphatase $>1.5 \mathrm{x}$ upper limit of normal [ULN]; or ALT $>2.5 \times$ ULN) or other hepatic abnormalities that in the opinion of the investigator would preclude the subject from participation in the study; patients with any chronic inflammatory autoimmune diseases; patients undergoing periodontal treatment in the last 3 months; women with positive pregnancy test or is known to be pregnant or lactating; patients who have had antibiotics in the last 3 months.

\section{Dental procedures}

At the beginning of the study a medical record of each patient was entered. Patients were examined using sterile equipment such as a mouth mirror, an explorer $\mathrm{n}^{\circ} 23$ and a WHO probe. There was no prior antibiotic prophylaxis. Patients were asked not to brush their teeth on the dental evaluation day, but they could use mouthwashes. Oral hygiene and periodontal status were assessed in every tooth, except for third molars, using Silness and Löe plaque index (PI) and gingival index (GI) (1964), ${ }^{22}$ measuring probing depth (PD) and clinical attachment loss (CAL). Four smooth surfaces (vestibular, lingual/palatal, distal and mesial) of 6 representative teeth $(1.6 ; 2.1$; $2.4 ; 3.6 ; 4.1 ; 4.4)$ were examined. In the case of missing dental pieces, their adjacent tooth, which had the same features, was evaluated. The PI was classified as follows: $0=$ no biofilm; $1=$ no biofilm observed at first sight, but found using a probe on gingival margin; $2=$ biofilm observed at first sight; $3=$ abundant biofilm. The highest values measured were considered. The GI was classified as follows: $0=$ total absence of signs of inflammation; $1=$ a slight change in color and texture; $2=$ visible signs of inflammation and gingival margin bleeding tendency in contact with the probe, and $3=$ apparent inflammation with spontaneous bleeding or ulcerations. Periodontal disease spread (PD and $\mathrm{CAL}$ ) was defined according to the percentage of deep pockets. 
The mean highest values of PD and CAL for each patient were determined through the division of the deepest pockets values by the total number of deep pockets. Periodontal disease (POD) was defined as the presence of four or more teeth with the highest value of PPD $\geq 3$ $\mathrm{mm}$ and $\mathrm{CAL} \geq 3 \mathrm{~mm} \cdot{ }^{22}$ Blood count: automated biochemical analysis systems (Wiener Lab CB 350i and Wiener Lab CM250, Wiener Lab SAIC, Riobamba 2944, Rosario, Argentina) and hematology analysis systems were used. Not all the patients were in a fasted state before blood sampling. Plasma concentrations:

a. Glycemia, total cholesterol.

b. White blood cells, hematocrit, hemoglobin and platelets.

c. CK-MB.

Reference values: CK-MB: $<25$ IU/L; hemoglobin: men 12.5-17 g $\%$ - women $11.5-15 \mathrm{~g} \mathrm{\%}$; platelet count: $150000-400000 / \mathrm{mm}^{3}$; plasma glucose: $70-100 \mathrm{mg} / \mathrm{dL}$; white blood cell count: men $5000-10000 /$ $\mathrm{mm}^{3}$ - women $4000-8000 / \mathrm{mm}^{3}$; total cholesterol $<200 \mathrm{mg} / \mathrm{dL}$.

\section{Statistical analysis}

In order to determine the sample size, the prevalence of periodontal disease was considered to be two times higher in the case group than in the control group, with an alpha error below 0.05 and a power of $80 \%$. Thus, the minimum number of subjects was 56 , 28 per group (assuming at least a 1:1 case-control ratio). The mean values were calculated through a cumulative sum, and to compare those nonparametric statistics was used: Mann-Whitney U test, given the number of patients per group. The categorical or dichotomous variables are presented as proportions and compared through $\mathrm{X}^{2}$ test or the Mantel-Haenszel test when necessary. For the univariate analysis of risk factors between groups, unadjusted odds ratio (OR) and $95 \%$ $\mathrm{CI}$ were used. If significant differences were found in the univariate analysis, the multinomial logistic regression would be applied. A significance level of $\mathrm{p}<0.05$ for a two-sided test was assumed. IBM SPSS statistics 22 was used for the statistical analysis.

\section{Results}

Table 1 shows the basal characteristics of both groups. A total number of 62 subjects were included: 30 cases and 32 controls. There were 28 men (93\%) in the case group and 30 (93.75\%) in the control group. In the case group, acute vascular events followed acute coronary syndrome (28 subjects) and acute peripheral artery disease (1 subject); 1 patient had posterior circulation ischemic stroke. The average ages were $60.10 \pm 8.93$ for cases and $60.53 \pm 8.97$ for controls $(\mathrm{p}=0.85)$. The $40 \%$ of cases $(\mathrm{n}=12)$ and $19 \%$ of controls $(\mathrm{n}=6)$ had smoking habits, this difference not being significant $(\mathrm{p}=0.15)$. In both groups, the prevalence of the other risk factors was similar: hypertension $(p=0.77)$, dyslipidemia $(p=0.45)$, diabetes $(p=0.59)$, sedentary lifestyle $(\mathrm{p}=0.99)$, former smoking habit $(\mathrm{p}=0.99)$, BMI $(p=0.76)$, number of teeth $(p=0.54)$, physical activity $(p=0.54)$. The combination of risk factors in both groups was highly frequent with no significant difference; 26 cases (86\%) and 28 controls ( $78 \%$ ) were exposed to at least 2 risk factors (OR: $0.92 ; 95 \% \mathrm{CI}: 0.21-4.10 ; \mathrm{p}=1$ ). The most common risk factor association in both groups was between hypertension and overweight/obesity. The proportion of patients with POD was considerably higher in the case group than in the control one $(p=0.02)$. For this association, the phi coefficient was +0.29 . The most marked difference was observed in patients with moderate POD $(p=0.05)$; regarding severe periodontitis, cases showed a slightly higher tendency than controls $(\mathrm{p}=0.07)$, and no notable differences were perceived in patients with mild POD $(p=0.31)$. Presence of gingivitis was equivalent in both groups $(\mathrm{p}=0.99)$. However, the plaque and gingival indexes were significantly higher in cases (PI: 1.74 \pm 0.64 vs. $0.99 \pm 0.69$ respectively; $\mathrm{p}<0.001$; GI: $1.61 \pm 0.66$ vs. $0.98 \pm 0.55$, respectively; $\mathrm{p}=0.001$ ) (Table 2 ). Among the cases, the number of affected blood vessels was: 1 vessel $=36.7 \%$; 2 vessels $=20 \%$, and 3 vessels $=33.3 \%$. When biochemical and hematological variables were analyzed, only one significant and expected difference was found between the two groups: CK-MB plasma levels ( $p=0.007)$. A tendency to higher plasma glucose levels $(\mathrm{mg} / \mathrm{dL})$ was observed in cases ( $\mathrm{p}=0.053)$, whereas no important differences were found in total cholesterol, white blood cell count, platelet count and hemoglobin level, as shown in Table 3.

Table I Baselines at both groups

\begin{tabular}{lllll}
\hline Variables & Cases n (\%) & $\begin{array}{l}\text { Controls } \\
\mathbf{n}(\%)\end{array}$ & OR (Cl95\%) & p $^{*}$ \\
\hline $\begin{array}{l}\text { Age } \\
\text { (years) }\end{array}$ & $60,10 \pm 8,93$ & $60,53 \pm 8,97$ & 0,87 \\
NofT & $18,13 \pm 6,86$ & $22,38 \pm 6,27$ & & 0,54 \\
HT & $17(56 \%)$ & $17(53 \%)$ & $\mathrm{I}, 15(0,42-3,14)$ & 0,77 \\
DLP & $12(40 \%)$ & $16(50 \%)$ & $0,66(0,24-1,82)$ & 0,45 \\
Dbt & $12(40 \%)$ & $10(31 \%)$ & $1,46(0,51-4,17)$ & 0,59 \\
Sed & $15(50 \%)$ & $16(50 \%)$ & $1(0,36-2,70)$ & 0,99 \\
Tbq & $12(40 \%)$ & $6(19 \%)$ & $2,66(0,84-8,46)$ & 0,15 \\
TbqR & $12(40 \%)$ & $12(37 \%)$ & $1,11(0,39-3,08)$ & 0,99 \\
FH & $8(26 \%)$ & $6(19 \%)$ & $I, 57(0.47-5,23)$ & 0.54 \\
BMI Kg/m3 & $27,31 \pm 4,99$ & $27,79 \pm 6,23$ & & 0,14 \\
\hline
\end{tabular}

NofT, number of teeeth; HT, hypertension; DLP, dyslipidemia; Dbt, diabetes mellitus; Sed, sedentary lifestyle; Tbq, current tabaquism; TbqR, remote tabaquism; BMI, body mass index; $\mathrm{FH}$, family history of acute ischemic heart disease

*Se utilizó prueba $U$ de Mann-Whitney para muestras independientes en la inferencia con variables continuas y prueba de $\chi 2$ para variables categóricas.

Table 2 Periodontitis between groups

\begin{tabular}{lllll} 
Variables & $\begin{array}{l}\text { Cases } \mathbf{n} \\
(\%)\end{array}$ & $\begin{array}{l}\text { Controls } \\
\mathbf{n}(\%)\end{array}$ & OR (CI95\%) & p* $^{*}$ \\
\hline POD & $2 I(70 \%)$ & $13(40 \%)$ & $3,4 I(I, I 9-9,76)$ & 0,02 \\
Minus POD & $5(16 \%)$ & $8(25 \%)$ & $0,60(0,17-2,09$ & 0,53 \\
Moderate POD & $9(30 \%)$ & $3(9 \%)$ & $4, I 4(0,99-17,17)$ & 0,05 \\
Severe POD & $7(23 \%)$ & $2(6 \%)$ & $4,56(0,86-24,07)$ & 0,07 \\
Gingivitis & $26(86 \%)$ & $27(84 \%)$ & $1,20(0,29-4,98)$ & 0,99 \\
GI & $1,6 I \pm 0,66$ & $0,98 \pm 0,55$ & & $0,00 I$ \\
PI & $1,74 \pm 0,64$ & $0,99 \pm 0,69$ & & $<0,00 I$ \\
\hline
\end{tabular}

POD, periodontal disease; $\mathrm{Gl}$, gingival index; $\mathrm{PI}$, plaque index

*Mann-Whitney $U$ test was used for independent samples of continues variables and $\chi 2$ test for dicotomous variables 
Table 3 Biochemical and hematologic variables between groups

\begin{tabular}{|c|c|c|c|}
\hline Variables & Cases & Controls & $\mathbf{p}^{*}$ \\
\hline & Media(SD) & Media(SD) & \\
\hline $\begin{array}{l}\text { Cholesterol } \\
(\mathrm{mg} / \mathrm{dl})\end{array}$ & $161,37 \pm 41,15$ & $188,97 \pm 47,29$ & 0,22 \\
\hline $\begin{array}{l}\text { Glucose } \\
(\mathrm{mg} / \mathrm{dl})\end{array}$ & $\mid 40,80 \pm 57,263$ & || $6,03 \pm 35,7 \mid 3$ & 0,053 \\
\hline $\begin{array}{l}\text { Leucocytes } \\
\text { count } \\
(\mathrm{mm} 3)\end{array}$ & $938|, 03 \pm 3| 7 \mid, 80$ & $8573,40 \pm 3040,49$ & 0,31 \\
\hline $\begin{array}{l}\text { Hemoglobin } \\
(\mathrm{g} \%)\end{array}$ & $13,64 \pm 2,08$ & $13,58 \pm 1,74$ & 0,90 \\
\hline $\begin{array}{l}\text { CK MB } \\
(\mathrm{UI} / \mathrm{I})\end{array}$ & $29,87 \pm 35,86$ & ||$, 8 \mid \pm 3,48$ & 0,001 \\
\hline $\begin{array}{l}\text { Uric Acid } \\
\text { (mg/dl) }\end{array}$ & $4,60 \pm 1,479$ & $5,65 \pm 1,748$ & 0,15 \\
\hline $\begin{array}{l}\text { Count } \\
\text { Plaques } \\
(\mathrm{mm} 3)\end{array}$ & $234833,33 \pm 126169,859$ & $213225,81 \pm 67325,928$ & 0,40 \\
\hline MPV (fl) & $8,29 \pm 1,66$ & $7,96 \pm 1,50$ & 0,42 \\
\hline
\end{tabular}

CK MB, creatinephosphokinase MB;VPM, mean platelet volume

*Mann-Whitney U-test was used for independent samples

\section{Discussion}

The systemic effects of periodontal disease have been extensively studied. Microorganisms that cause this condition, interleukins, cross-reacting antibodies, etc. are involved; ${ }^{15-26}$ something similar happens with the physiopathogenesis of atheromatous disease. ${ }^{10}$ It is necessary to demonstrate how these two diseases interact in order to study the clinical impact and the prognosis. This study shows a strong association between POD and vascular disease, defined as acute vascular events (mostly acute coronary syndrome). The final results indicate that POD was more prevalent in subjects with AVE than in those who did not suffer from this condition: patients with acute vascular events had 3.41 more chance of being affected by them if they also had periodontitis. On the hand, plaque and gingival indexes, which do not determine the periodontal status but represent a greater risk of getting the disease, were considerably higher in the control group. With the aim of reducing possible biases, controls were screened paying attention to potentially misleading factors such as contemporaneous inclusion of controls, recruitment in the same institution, age, gender, BMI and other common risk factors for vascular disease. ${ }^{12}$ Since the basal characteristics of both groups showed no significant differences, the groups were comparable. Given the variety of risk factors and the fact that most subjects were exposed to more than one, we selected the three best predictors of vascular events: hypertension, diabetes and smoking. ${ }^{19}$ We used the conventional definitions of POD to make the results of this study compatible with those of future studies on the same topic. ${ }^{25}$ The results of this study are similar to those found in previous articles showing a clear relation between these two conditions. However, they display a bias in their design, which makes their conclusions weak. ${ }^{27,28}$ Another study showed opposite conclusions, not finding convincing evidence of a causal relationship between POD and coronary artery disease (HR: 1.14; 95 \% CI: 0.96-1.36). It was a prospective cohort study with patients suffering from POD and subjects with a healthy periodontal status. The follow-up was conducted in different years for a short period of time (2-4 years) to assess the possible development of vascular events after study inclusion. In the end, the study had not been designed to prove this association. ${ }^{29}$ The first study with a case-control design (1989), where the risk factors considered were age, cholesterol level, smoking and diabetes, shows a clear relationship between these two conditions. ${ }^{30}$. The findings in a study published in our country also showed a strong association between POD and vascular disease/ events; the odds ratio was similar to ours (OR: 3.26; 95 \% CI: 1.19 9.76). A case-control design was used and the risk factors included were diabetes, hypertension, obesity, dyslipidemia, current and former smoking. However, the differences between the case group and the control group are not clear since they are not detailed in the results; the number of patients expressed in the aim section does not match that in the results, making it difficult to interpret the findings (31). Something similar happened with two systematic reviews which included different designs such as cross-sectional and case-control studies. ${ }^{13-31}$ Our project, besides including matching risk factors mentioned in previous articles, incorporated vascular disease history in first degree relatives, sedentary lifestyle, number of teeth and BMI. In conclusion, this study demonstrates a strong association between periodontal disease and acute vascular events, the former having an even greater impact than traditional risk factors. We consider that the best design to prove this association is still the case-control one, given that cohort studies should have a longer follow-up period that would lead to changes in the patients' periodontal statuses and, therefore, to different findings and so on. Having said this, when choosing a case-control design, the difficulty lies in screening controls properly, establishing endpoints and calculating the sample size, all of which makes conclusions more valid.

\section{Acknowledgments}

None.

\section{Conflicts of interest}

The author declares that there is no conflict of interest

\section{Funding}

Medical Foundation of Río Negro and Neuquén.

\section{References}

1. Lockhart PB, Bolger AF, Papapanou PN, et al. Periodontal Disease and Atherosclerotic Vascular Disease: Does the Evidence Support an Independent Association? A Scientific Statement from the American Heart Association. Circulation. 2012;125(20):2520-2544.

2. Nair P. Pathogenesis of Apical Periodontitis and the Causes of Endodontic Failures. Crit Rev Oral Biol Med. 2004;15(6):348-381.

3. Ramfjord S, Ash M. Periodontology and Periodontics., USA: WB Saunders Company; 1979. 1-600 p.

4. Chakrapani S, Sirisha K, Srilalitha A, et al. Choice of diagnostic and therapeutic imaging in periodontics and implantology. J Indian Soc Periodontol. 1979;17(6):711-718.

5. Khosravi R, Ka K, Huang T, et al. Tumor necrosis factor- $\alpha$ and interleukin-6: potential interorgan inflammatory mediators contributing to destructive periodontal disease in obesity or metabolic syndrome. Mediators of inflammation, Mediators Inflamm. 2013;728987.

6. Olavegogeascoechea PA, Brusca MI, Noier M, et al. Pro-Inflammatory Cytokine Concentration in Gingival Fluid in Obese. J Dent Health Oral Disord Ther. 2013;5(7):00180. 
7. Dominguez K, Penman-Aguilar A, Man-Huei Ch, et al. Vital Signs: Leading Causes of Death, Prevalence of Diseases and Risk Factors, and Use of Health Services among Hispanics in the United States-2009-2013. MMWR Morbidity and Mortality Weekly Report. 2015.

8. Bhatt DL, Steg PG, Ohman EM, et al. International Prevalence, Recognition, and Treatment of Cardiovascular Risk Factors in Outpatients With Atherothrombosis. JAMA. 2006;295(2):180-189.

9. Libby P, Ridker PM, Maseri A. Inflammation and Atherosclerosis Circulation. 2002;105(9):1135-1143.

10. Ross R. Atherosclerosis-An Inflammatory Disease. $N$ Engl J Med. 1999;340(2):115-126.

11. Kannel WB. Factors of Risk in the Development of Coronary Heart Disease-Six-Year Follow-up Experience: The Framingham Study. Ann Intern Med. 1961;55(1):33.

12. Bahekar AA, Singh S, Saha S, et al. The prevalence and incidence of coronary heart disease is significantly increased in periodontitis: A metaanalysis. Am Heart J. 2007;154(5):830-837.

13. Friedewald VE, Komman KS, Beck JD, et al. The American Journa of Cardiology and Journal of Periodontology Editors' Consensus: Periodontitis and Atherosclerotic Cardiovascular Disease. Am J Cardiol. 2009;104(1):59-68.

14. Alonso-Gonzalez R, Perez-Hernandez A, Silvestre-Rangil J, et al Cardiovascular disease versus periodontal disease: chronic systemic infection as a link. J Clin Exp Dent. 2011;(5):e476-e482.

15. Nakajima T, Honda $\mathrm{T}$, Domon $\mathrm{H}$, et al. Periodontitis-associated upregulation of systemic inflammatory mediator level may increase the risk of coronary heart disease. J Periodontal Res. 2010;45(1):116-122.

16. Nakib SA, Pankow JS, Beck JD, et al. Periodontitis and Coronary Artery Calcification: The Atherosclerosis Risk in Communities (ARIC) Study. $J$ Periodontol. 2004;75(4):505-510.

17. Akinkugbe AA, Saraiya VM, Preisser JS, et al. Bias in estimating the cross-sectional smoking, alcohol, obesity and diabetes associations with moderate-severe periodontitis in the Atherosclerosis Risk in Communities study: comparison of full versus partial-mouth estimates. $J$ Clin Periodontol. 2015;2(7):609-621.

18. Lopez R, Scheutz F, Errboe M, Baelum V. Selection bias in casecontrol studies on periodontitis: a systematic review. Eur J Oral Sci. 2007;115(5):339-343.
19. Grundy SM, Pasternak R, Greenland P, et al. Assessment of Cardiovascular Risk by Use of Multiple-Risk-Factor Assessment Equations. Circulation. 1999;100:1481-1492.

20. Amsterdam EA, Wenger NK, Brindis RG, et al. 2014 AHA/ACC guideline for the management of patients with non-ST-elevation acute coronary syndromes. Circulation. 2014;130(25):2354-2394.

21. Brown W, Trost S, Bauman A, Mummery K, et al. Test-retest reliability of four physical activity measures used in population surveys. J Sci Med Sport. 2004;7(2):205-215.

22. Silness J, Löe H. Periodontal Disease in Pregnancy II. Correlation Between Oral Hygiene and Periodontal Condition. Acta Odontol Scand. 1964;22(1):121-135

23. Brown L, Brunelle J, Kingman A. Periodontal status in the United States, 1988-1991: Prevalence, extent, and demographic variation. J Dent Res. 2010;45(1):116-122.

24. Beck JD, Offenbacher S. Systemic Effects of Periodontitis: Epidemiology of Periodontal Disease and Cardiovascular Disease. J Periodontol. 2005;76(11 Suppl):2089-2100.

25. Cekici A, Kantarci A, Hasturk H, et al. Inflammatory and immune pathways in the pathogenesis of periodontal disease. Periodontol. 2013;2000 64(1):57-80.

26. Johansson CS, Richter A, Lundström Å, et al. Periodontal conditions in patients with coronary heart disease: a case-control study. J Clin Periodontol. 2008;35(3):199-205

27. Amabile N, Susini G, Pettenati-Soubayroux I, et al. Severity of periodontal disease correlates to inflammatory systemic status and independently predicts the presence and angiographic extent of stable coronary artery disease. J Intern Med. 2008;263(6):644-652.

28. Hujoel PP, Drangsholt M, Spiekerman C. Periodontal Disease and Coronary Heart Disease Risk. JAMA. 2000;284(11):1406.

29. Mattila K, Nieminen M, Valtonen VV, et al. Association between dental health and acute myocardial infarction. BMJ. 1989;98(6676):779-781.

30. Nicolisi L, Lewin P, Díaz Casale A. La enfermedad periodontal como factor de riesgo en la cardiopatía isquémica. Rev Argen Cardiol. $2003 ; 71: 250-255$

31. Humphrey LL, Fu R, Buckley DI, et al. Periodontal Disease and Coronary Heart Disease Incidence: A Systematic Review and Meta-analysis. J Gen Intern Med. 2008;23(12):2079-2086. 